\title{
Contar historias con los datos: Aragón Open Data Focus, una experiencia innovadora de reutilización de los datos del sector público
}

\author{
Data storytelling: Aragón Open Data Focus, an innovative experience of reusing public sector data
}

\author{
Lorea Akerreta Escribano (1), Julián Moyano Collado (2)
}

(1) Deloitte, Plaza del Castillo, 21, 2 31008 Pamplona, Navarra (España), lakerreta@deloitte.es.

(2) Gobierno de Aragón, Edificio Pignatelli, Paseo María de Agustín, 36, 50071 Zaragoza (España), jmoyano@aragon.es

\begin{abstract}
Resumen
En los últimos años, las Administraciones Públicas han realizado, en un ejercicio de transparencia, un importante esfuerzo por abrir sus datos para que, a través de la reutilización de esta información, pueda generarse un impacto y valor real sobre el territorio. Sin embargo, en muchas ocasiones su complejidad hace que la ciudadanía no perciba los beneficios de su uso y la utilice. En este escenario, los avances tecnológicos y una nueva cultura de consumo de la información derivada de la democratización en su acceso han sentado las bases del desarrollo del periodismo de datos. Aragón Open Data, la apuesta de Gobierno de Aragón por los datos abiertos, ha desarrollado durante 2020 un nuevo servicio que busca unir estos dos factores con el objetivo de incentivar la reutilización de los datos del sector público, hacer pedagogía sobre sus posibilidades y empoderar a los distintos perfiles de usuarios en el uso de los datos públicos. El resultado del proyecto, enmarcado en su estrategia 2019-2022 y cofinanciado por el Fondo Europeo de Desarrollo Regional (FEDER) de la Unión Europea, es Aragón Open Data Focus, un servicio gratuito que permite a los usuarios del portal desarrollar y compartir sus propias historias a partir de los datos abiertos de Aragón.
\end{abstract}

Palabras clave: Datos abiertos. Data storytelling. Transparencia. Reutilización de la información. Innovación pública. Aragón.

\section{Introducción}

La Open Knowledge Foundation (2012), fundación sin ánimo de lucro creada en 2004 para apoyar la difusión de conocimiento abierto en sentido amplio, define los datos abiertos como

[...] datos que pueden ser utilizados, reutilizados y redistribuidos libremente por cualquier persona, y que se encuentran sujetos, cuando más, al requerimiento de atribución y de compartirse de la misma manera en que aparecen.

En el contexto de la tendencia observada en los últimos años hacia modelos de gobierno abierto, más transparentes, participativos y orientados a la rendición de cuentas a la ciudadanía, los datos

\begin{abstract}
In recent years, Public Administrations have made, in an exercise of transparency, an important effort to open their data with the aim that, through the reuse of this information, a real impact and value can be generated on the territory. However, on many occasions the complexity of the matter means that citizens are not able to perceive the benefits that can be derived from its use, and they distance themselves from the use of open data. In this same scenario, technological advances and a new culture of information consumption derived from the democratisation of its access have laid the foundations for the development of data journalism. Aragón Open Data, the Government of Aragon's commitment to open data, has developed a new service during 2020 that seeks to unite these two factors with the aim of encouraging the reuse of public sector data, educating about its possibilities and empowering the different user profiles in the use of public data. The result of the project, framed in its 2019-2022 strategy and co-financed by the European Regional Development Fund (ERDF) of the European Union, is Aragón Open Data Focus, a free service that allows users of the portal to develop and share their own stories based on Aragon's open data.
\end{abstract}

Keywords: Open data. Data storytelling. Transparency. Information reuse. Public innovation. Aragon.

abiertos han ido escalando en términos de importancia como uno de los elementos nucleares de este tipo de políticas. Los distintos gobiernos han invertido una importante cantidad de recursos en abrir la información de la que disponen para que cualquier persona interesada pueda acceder a ella y reutilizarla con la única obligación de reconocer su autoría y citar al organismo publicador como fuente de dichos datos. El caso de España es especialmente notable, situándose año tras año en las primeras posiciones de la Unión Europea en los estudios de madurez de apertura de datos del EU Open Data Portal en los cuatro indicadores observados: política, portal web, impacto y calidad. 
Sin embargo, este esfuerzo por parte de las Administraciones Públicas se ha visto, en la práctica, poco correspondido por los usuarios. Con la excepción de colectivos específicos de usuarios, como programadores y desarrolladores, en general se desconoce la existencia de estas iniciativas o no se hace uso de ellas por desinterés en la información publicada, percepción de complejidad o, simplemente, porque no se aprecia el valor de la reutilización de estos datos.

En este sentido, algunas Administraciones Públicas se encuentran inmersas en la búsqueda de soluciones o alternativas que les permitan afrontar el reto de acercar los datos abiertos al común de la ciudadanía y facilitar su reutilización. Este es el caso del Gobierno de Aragón, que en su Estrategia Aragón Open Data 2019-2022 (Gobierno de Aragón, 2019) establece entre sus siete ejes de actuación el potenciar la implicación de los usuarios del portal e incluir nuevos servicios de valor añadido.

Bajo esta premisa, la Dirección General de Administración Electrónica y Sociedad de la Información de Gobierno de Aragón, responsable de la administración del portal autonómico de datos abiertos, llevó a cabo un proceso de reflexión interno en el que, mediante la observación de experiencias de éxito a nivel internacional en materia de reutilización de la información pública, identificó como buena práctica capaz de generar un retorno de valor real la puesta a disposición de un servicio que permitiera que usuarios sin conocimientos técnicos pudieran manipular los datos abiertos disponibles para generar visualizaciones que pudieran emplearse, a su vez, para construir historias en las que se interpretaran estos. Los beneficios de un servicio de este tipo van desde el empoderamiento de la ciudadanía al facilitar su acceso a la información pública hasta reforzar la transparencia de la actuación pública, pasando por el establecimiento de un marco de diálogo directo con distintos tipos de usuarios que permita mejorar, además, la calidad del servicio prestado.

Así, en el año 2020, el Gobierno de Aragón se puso manos a la obra y lanzó el proyecto Aragón Open Data Focus, nombre elegido para el nuevo servicio del portal de datos abiertos de Aragón. El trabajo desarrollado partió de un análisis de los datos disponibles y la caracterización de los potenciales colectivos de usuarios que podrían hacer uso de estos datos en función de su conocimiento de los datos abiertos, su actitud hacia los mismos o los temas que pudieran resultarles de interés. Tras este análisis, se abrió un proceso de diálogo, condicionado por la pandemia de la COVID-19, con los distintos colectivos definidos.

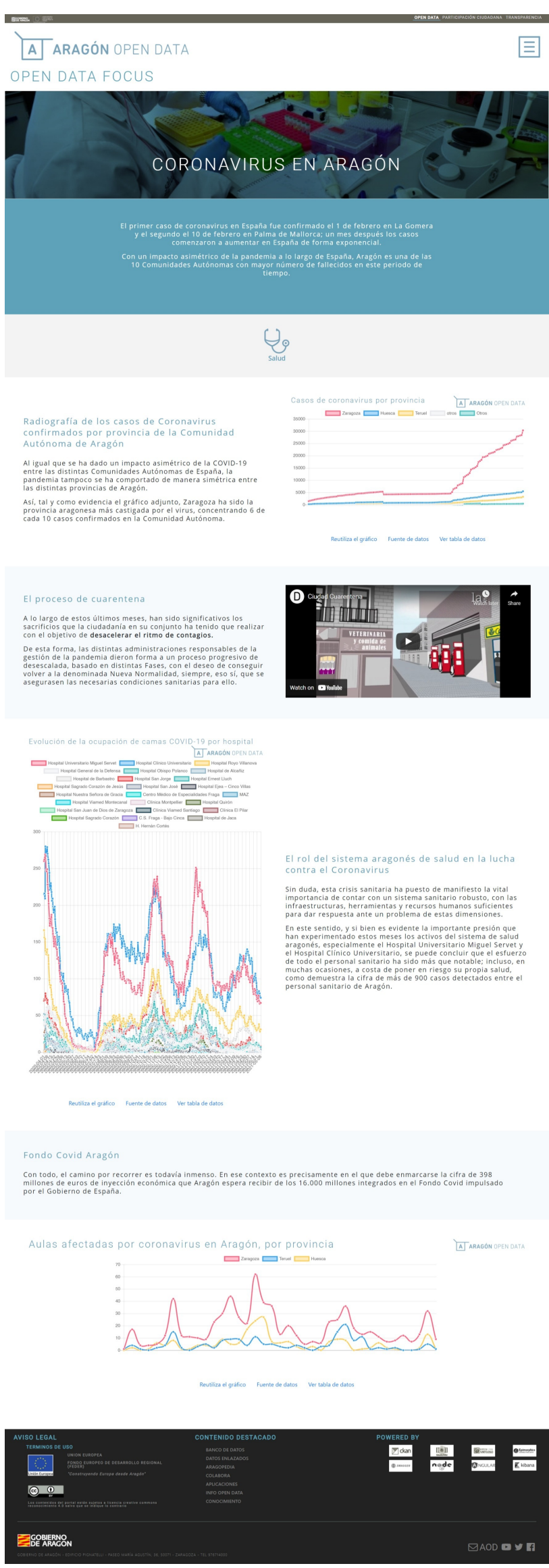

Figura 1. Historia "Coronavirus en Aragón" 
En este proceso participaron casi 300 personas a través de distintos eventos web en los que se daba a conocer el proyecto de Aragón, se presentaban distintos casos de interés adaptados a la caracterización de cada colectivo, se abría un debate con los participantes sobre los datos que les resultaban de mayor interés y se identificaban los aspectos de mejora que les facilitarían reutilizar la información disponible.

El resultado de este trabajo ha sido la creación de un servicio web que permite a los usuarios del portal de Aragón convertirse en "periodistas de datos", utilizando los más de 5.000 recursos disponibles en Aragón Open Data para la construcción de historias de su interés y consultar las generadas por otros usuarios. Así, a través del servicio, los usuarios pueden construir, de una forma sencilla, visualizaciones con los datos del catálogo, acompañarlas de recursos adicionales (como vídeos, imágenes o presentaciones) y redactar sus propios artículos con base en la información disponible. La Figura 1 muestra una de las historias creadas en el nuevo servicio que explica cómo ha impactado la pandemia de coronavirus en Aragón, a través de la presentación gráfica de los casos registrados por provincia, la evolución de la ocupación de camas COVID-19 por hospital y las aulas afectadas por provincia, además de un vídeo que explica el proceso de cuarentena para evitar la expansión del virus.

El presente artículo pretende presentar el proyecto desarrollado desde Gobierno de Aragón, profundizando en el potencial de los datos abiertos para crear valor, presentando las conclusiones obtenidas del trabajo desarrollado con los distintos colectivos y explicando el funcionamiento del nuevo servicio, poniendo en valor esta experiencia pionera en España y abriendo la puerta a nuevos modelos de innovación en el acercamiento de los datos abiertos a la ciudadanía. Para ello, se llevará a cabo una breve presentación del contexto actual de los datos abiertos en el sector público y su contribución al desarrollo económico, la mejora de los servicios públicos y el empoderamiento de la ciudadanía por parte de los gobiernos abiertos, seguida de una explicación del proyecto Aragón Open Data Focus y los resultados alcanzados, para finalizar con las principales conclusiones obtenidas de los trabajos desplegados.

\section{Contexto}

2.1. Antecedentes: la transparencia de las Administraciones públicas

La pérdida de confianza de la ciudadanía en las instituciones públicas y sus gestores ha supuesto un catalizador para la creación de nuevos mecanismos de relación que permitan a la ciudadanía conocer cómo se toman las decisiones, cómo se manejan y gestionan los fondos públicos o cuáles son los criterios que rigen la actuación de los gobiernos (Cerrillo i Martínez, 2011).

En este contexto, la transparencia, frente a la idea de secretismo y de reserva de la información, se erige como una herramienta clave para asegurar la higiene democrática, así como la participación e involucración de la ciudadanía en la gestión de lo público, constituyéndose como una de las reglas de la legitimación real y social del comportamiento de los gobiernos, instituciones públicas y clase política (Cordero, 2007).

La normativa, tanto de carácter internacional, como a nivel estatal, avala y aboga por una transparencia real de las Administraciones públicas. Así, la Carta de Derechos Fundamentales de la Unión Europea, en sus artículos 41 y 42, reconoce el derecho de los ciudadanos europeos a una buena administración y a acceder libremente a los documentos contenidos en las instituciones europeas (Palomares, 2017), sentando las bases para el desarrollo normativo de este principio.

Asimismo, el Convenio 205 del Consejo de Europa sobre el Acceso a los Documentos Públicos, de 18 de junio de 2009, reconoce la importancia de la transparencia de autoridades públicas en una sociedad democrática y plural (Montero, 2020), considerando que el ejercicio del derecho de acceso a los documentos públicos proporciona una fuente de información para los distintos tipos de agentes que viven y operan en los territorios; ayuda al público a formarse una opinión sobre el estado de la sociedad y sobre la labor de la Administración; y fomenta la integridad, la eficacia, la eficiencia y la responsabilidad de autoridades públicas, ayudando así a que se afirme su legitimidad. Considera, por tanto, que todos los documentos generados en el ejercicio de las competencias de la Administración son en principio públicos y solamente pueden ser retenidos para proteger otros derechos e intereses legítimos.

En el ámbito estatal y con carácter básico, se aprobó la Ley 19/2013, de 9 de diciembre, de transparencia, de acceso a la información pública y buen gobierno. Su aplicación y contenido se vertebra en tres vertientes: la transparencia en la actividad pública a través de la publicidad activa; el reconocimiento y la garantía del derecho de acceso a la información pública; y el buen gobierno, que se concreta en obligaciones que vinculan a los responsables públicos y en las consecuencias que derivan de su vulneración (Cotino, 2015). 
Así, si bien es cierto que existe una norma jurídica que obliga a la transparencia de la información por parte de las Administraciones públicas, las mismas están aprovechando las nuevas tecnologías para incrementar exponencialmente la difusión pública de toda la información de interés general que poseen las entidades que se financian con los impuestos de los ciudadanos, yendo más allá, buscando fórmulas que les permitan configurar gobiernos más abiertos y accesibles (Blanes, 2013).

\subsection{La reutilización de la información pública como materialización del concepto de Gobierno Abierto}

Consecuencia de la evolución de las relaciones con la ciudadanía, emerge el concepto de Gobierno Abierto, que adquiere carta de naturaleza institucional en 2009 con la aprobación de una directiva de gobierno abierto, la Open Government Directive (Obama, 2009), por la Administración Obama en Estados Unidos, como un nuevo enfoque aglutinador de esfuerzos por mejorar las capacidades del gobierno y mejorar las administraciones públicas (Conejero, 2013), estableciéndose como una tipología de gobernanza pública basada en los principios de transparencia, colaboración y participación. Se trata de un modelo de gobernanza que apuesta por el diálogo y el equilibrio de poder entre gobierno y gobernados, al mismo tiempo que aboga por la corresponsabilidad ciudadana en la gestión pública (Clabo y Ramos-Vielba, 2015).

En este escenario aparece la apuesta de los gobiernos por los datos abiertos como un nuevo camino a explorar en las relaciones con la ciudadanía. Se distinguen de la transparencia en que mientras que esta consiste en la puesta a disposición de información relevante de una manera comprensible para asegurar que cumple su propósito divulgativo, los datos abiertos presentan la información de la manera menos tratada, en distintos formatos y con el mayor grado de desagregación disponible, de manera que puedan ser utilizados o reutilizados con otros propósitos que el meramente informativo, siempre con el único requisito de atribución de su fuente (Aleixandre-Benavent, Ferrer y Peset, 2019), contribuyendo a la satisfacción de distintas necesidades particulares de los usuarios.

En este contexto, el sector público produce una gran variedad de información que es potencialmente reutilizable, como puede ser la información social, económica, geográfica, estadística, etc. Esta información es muy atractiva para su reutilización ya que es completa, fiable y de calidad, estimando el European Data Portal, en su estudio The Economic Impact of Open Data: Opportunities for Value Creation in Europe (European Data Portal, 2020), que el tamaño de mercado para los datos abiertos tiene un valor de 184.000 millones de euros y que, para 2025, crecerá hasta una horquilla de entre 199.000 y 334.000 millones de euros. Así, a través de iniciativas de Open Data, se quiere lograr que la reutilización y redistribución de la información pública repercuta en beneficio tanto de las administraciones públicas (Abella, 2019), que sacan a la luz sus datos, como de los ciudadanos y empresas, que ven cubiertas algunas de sus necesidades reales.

Si bien existen numerosos ejemplos de las formas en que los datos abiertos ya están creando valor social y económico, aún se desconoce la amplitud completa de sus posibilidades a futuro. Las nuevas combinaciones de datos pueden crear nuevos conocimientos y perspectivas, que pueden conducir a campos de aplicación completamente nuevos. El pasado nos enseña, una vez más, que la combinación de datos nuevos da lugar a un conocimiento mayor: por ejemplo, cuando el Dr. John Snow descubrió la relación entre la contaminación del agua potable y el cólera en Londres en el siglo XIX al combinar datos sobre las muertes por cólera con la ubicación de los pozos de agua. Esto condujo a la construcción de los sistemas de alcantarillado de Londres y mejoró enormemente la salud general de la población. Desarrollos como este continuarán sucediendo a medida que fluyan conocimientos inesperados de la combinación de diferentes conjuntos de datos abiertos.

Este potencial sin explotar se puede desatar si los datos públicos del gobierno se convierten en datos abiertos. Sin embargo, esto solo sucederá si los datos son real y efectivamente abiertos, es decir, si no existen restricciones (legales, financieras o técnico-tecnológicas) para su reutilización por parte de otros usuarios: la existencia de cualquier obstáculo excluirá a las personas de reutilizar los datos públicos y dificultará la búsqueda de formas valiosas de hacerlo.

\subsection{La contribución al desarrollo económico y competitivo de los territorios, la mejora en la prestación de los servicios públicos y el empoderamiento de la ciudadanía por parte de los gobiernos abiertos}

Los datos abiertos contribuyen directamente al crecimiento de las economías y a la transformación de la sociedad, y buena prueba de ello es la apuesta e implicación de gobiernos de todo el mundo por la promoción de portales que hagan accesibles los datos públicos más allá de servir 
como tarjeta de presentación para exhibir la transparencia pública, de bajo coste y relativamente fácil de construir (Ramos, et al., 2012).

En el contexto de la innovación, la puesta a disposición de datos abiertos por parte de la Administración pública estimula que se descubran nuevas oportunidades de negocio para empresas y se eliminen barreras al acceso, uso e intercambio de datos, de manera que finalmente se contribuya al crecimiento económico (Attard, et al., 2015). Por ejemplo, en Islandia se publican con carácter anual datos correspondientes a la calidad de los corderos de las distintas granjas de cría existentes en el país. De esta manera, desde las instituciones públicas, se facilita a granjeros, agricultores u otros agentes de la cadena de valor, información que puede ser de su interés.

Asimismo, el hecho de poner en disposición de empresas los datos fomenta un mayor conocimiento, contribuyendo en última instancia a que las decisiones empresariales se tomen con mayor base, resultando estas más adecuadas (Kucera y Chlapek, 2014).

A nivel gubernamental, los datos abiertos contribuyen a que los gobiernos puedan equilibrar mejor la prestación de servicios, abordando problemas como el tráfico, la seguridad, la movilidad en las ciudades $u$ otras cuestiones, como la salud o la igualdad, transformando el modelo de relación con el ciudadano, creando sociedades más avanzadas y participativas, y fomentando la colaboración público-privada. Por ejemplo, en Reino Unido no existe una obligación legal para las autoridades locales de ofrecer baños públicos o información sobre sus equipamientos, localizaciones $u$ horarios, lo que puede impactar sobre la igualdad o diversidad de la sociedad: un estudio de 2003 identificó que muchas mujeres planificaban sus desplazamientos diarios en función de los aseos públicos disponibles y conocidos (Greed, 2003). A esta circunstancia se sumó la aprobación en 2004 de la Disability Discrimination Act (DDA), que reconoció la incontinencia como una discapacidad. La solución a esta situación se articuló en 2011 a través del Great British Public Toilet Map, una iniciativa público-privada basada en datos abiertos que ofrece información sobre los aseos públicos existentes, mejorando la calidad de vida de la ciudadanía, especialmente entre la población de mayor edad (Bichard y Knight, 2012).

Por otra parte, la existencia de datos abiertos permite a los gobiernos tener un mayor conocimiento de la estructura de coste de gobiernos de características similares, de empresas, etc., con- tribuyendo a una mejor gestión de los fondos públicos. Al respecto, el estudio del European Data Portal The Economic Impact of Open Data: Opportunities for value creation in Europe (European Data Portal, 2020a), cifra el ahorro en costes para la Administración Pública en 2020 en 1.700 millones de euros.

Finalmente, los datos abiertos también permiten el desarrollo de nuevos servicios innovadores. Un ejemplo ilustrativo es la experiencia Desafío modal, en México, en la que se simulan situaciones de traslado de un origen a un destino con diversas formas de locomoción y diferentes rutas a través de la reutilización de datos abiertos. Los resultados de esta simulación son, cuanto menos, curiosos: en hora punta, bicicletas, patines y motocicletas aparecen como los medios de transporte más eficientes, acumulando la bicicleta una diferencia de más de 24 minutos con el automóvil en Ciudad de México, o de 15 en el caso de Oaxaca (Sève y Redondo, 2019).

En el escenario descrito, el desarrollo de un modelo de compartición de datos y co-creación de servicios entre los sectores público y privado se constituye como una pieza clave de futuro (Cabezali, 2020). Así lo ha entendido la UE, que en su informe Analytical Report 12: Business-to-Government Data Sharing (European Data Portal, $2020 b)$ concluye que el suministro de datos desde entidades privadas a públicas (B2G) para su reutilización fomentará el crecimiento, el empleo y la competitividad europea ahorrando costes, tiempo y vidas, estableciendo en consecuencia un plan de 6 pasos para avanzar en este camino hacia la "quinta libertad": el movimiento libre de datos en el Mercado Digital Europeo.

Sin embargo, el despliegue de iniciativas de datos abiertos no está exenta de dificultades: así, actualmente, dos de los principales retos que enfrenta son, por un lado, la necesidad de desplegar una labor pedagógica con la ciudadanía y el tejido empresarial que les permita comprender el potencial de su uso; y, por otro, facilitar esa reutilización mediante la capacitación de los distintos grupos de agentes que viven y operan en los territorios (Álvarez et al., 2014).

\section{Objetivos del proyecto}

\subsection{La visión de los datos abiertos}

del Gobierno de Aragón: retos y respuesta

Aragón Open Data (1) es el medio a través del que se materializa el compromiso del Gobierno de Aragón de publicar sus datos de forma abierta. Este compromiso se origina en el ejercicio 2012 y tiene como objetivo fundamental la 
creación de riqueza económica a través de la reutilización de la información y el fomento de la transparencia, lo cual dota de un carácter particular al proyecto al constituirlo como un planteamiento más evolucionado del proceso de apertura de la información pública que busca, ya desde sus inicios, el fomento de la utilización de la misma para la creación de un valor tangible.

A lo largo de los años se han sucedido avances notables en el proyecto, administrado por la Dirección General de Administración Electrónica y Sociedad de la Información de Gobierno de Aragón, que han permitido situar al portal de datos abiertos de Aragón a la cabeza de las propuestas autonómicas en España, tal y como se muestra en la Tabla I.

\begin{tabular}{lrcc}
\hline Comunidad Autónoma & Datasets & API & Catál. RDF \\
\hline Andalucía & 555 & No & No \\
\hline Aragón & 2.823 & Sí & Sí \\
\hline Asturias & 24 & No & No \\
\hline Baleares & 182 & Sí & Sí \\
\hline Canarias & 186 & Sí & No \\
\hline Cantabria & 356 & Sí & No \\
\hline Castilla-La Mancha & 266 & No & No \\
\hline Castilla y León & 487 & No & No \\
\hline Cataluña & 577 & Sí & No \\
\hline Comunidad Valenciana & 492 & Sí & No \\
\hline Extremadura & 25 & No & No \\
\hline Galicia & 366 & No & Sí \\
\hline La Rioja & 318 & Sí & Sí \\
\hline Madrid & 432 & Sí & No \\
\hline Murcia & 765 & Sí & No \\
\hline Navarra & 1.380 & Sí & No \\
\hline País Vasco & 4.966 & Sí & No \\
\hline Tablal Comparatia & & & Nos
\end{tabular}

Tabla I. Comparativa entre los portales de datos autonómicos, 2020

Así, en 2020 era la segunda Comunidad en términos de conjuntos de datos abiertos publicados, destacando su banco de datos (2) como uno de sus activos más importantes. Para facilitar la búsqueda de información en un repositorio tan complejo y heterogéneo, cuenta con un avanzado sistema de búsqueda que permite tanto la búsqueda libre como el empleo de otros criterios, ya sean el número de descargas del recurso, la fecha de última actualización, temas, tipos de información, organización publicadora o etiquetas, entre otras.

La construcción de este banco de datos se ha materializado gracias a la implicación y compromiso de un amplio abanico de actores del sector público aragonés, que colaboran con el proyecto preparando y cediendo sus datos, desde 29 Direcciones Generales del gobierno autonómico (Administración Electrónica y Sociedad de la Información, Administración Local, Asistencia Sanitaria, Patrimonio y Organización, Cultura, Medio Natural y Gestión Forestal, etcétera) hasta la Universidad de Zaragoza, pasando por otras instituciones como los Institutos Aragoneses de Estadística, Empleo o del Agua, la Junta de Reclamaciones EconómicoAdministrativas de la Comunidad Autónoma de Aragón o Intervención General, entre otros. La variedad de publicadores enriquece las temáticas de información disponibles en el banco de datos, que pasan por cuestiones como ciencia y tecnología, comercio, cultura y ocio, demografía, deporte, economía, educación, empleo, energía, hacienda, industria, legislación y justicia, medio ambiente, medio rural, salud, sector público, seguridad, sociedad y bienestar, transporte, turismo o urbanismo e infraestructuras.

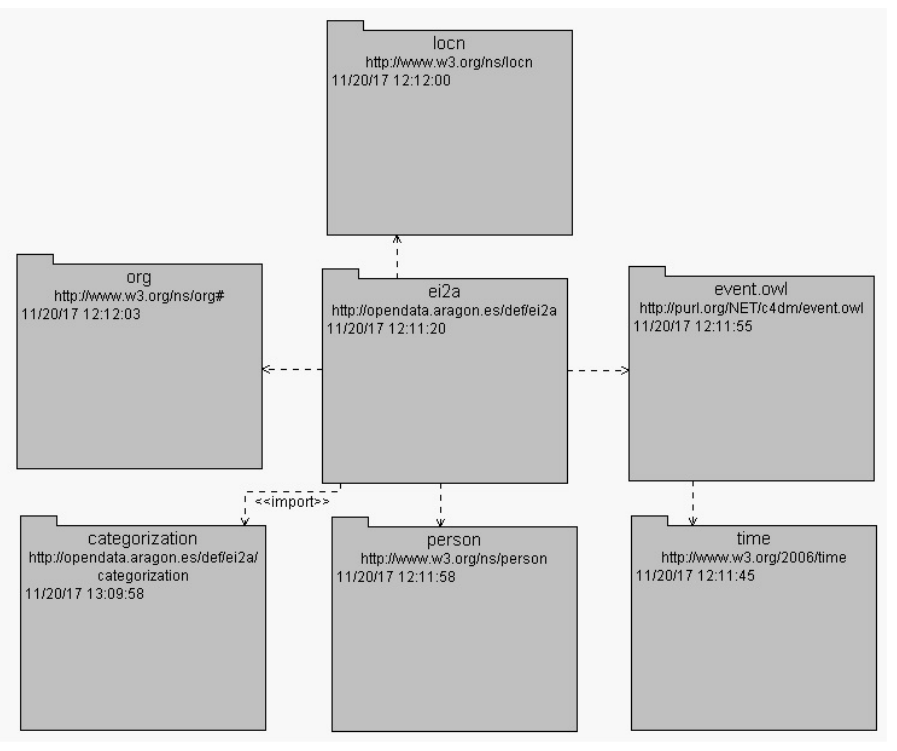

Figura 2. Ontologías y vocabularios usados para el desarrollo de la propuesta ontológica EI2A

Adicionalmente, Aragón Open Data ha desarrollado su propia ontología (EI2A, Estructura de Información Interoperable de Aragón), resumida en la Figura 2, que permite generar un lenguaje común entre las bases de datos y aplicaciones del Gobierno de Aragón, ha puesto a disposición de los usuarios del portal tres APIs adicionales a la nativa de CKAN (la aplicación web de código abierto que soporta el proyecto) para acceder a 
los datos de Aragón Open Data y ha incorporado un punto SPARQL de acceso a la información semántica, con su correspondiente información de consulta, facilitando el uso de los datos por parte de los usuarios técnicamente más avanzados.

En este contexto de desarrollo del proyecto, el Gobierno de Aragón aprobó, en el ejercicio 2019, la Estrategia Aragón Open Data 2019-2022 con la visión de que el portal de datos abiertos del Gobierno de Aragón se constituya en el portal de referencia en el ámbito de la gestión del dato abierto y proporcione un tratamiento de los sistemas de información que no sólo genere riqueza, sino que desarrolle y consolide, entre otros, el sector infomediario. Dicha estrategia analizaba, además, el mapa actual de agentes reutilizadores de datos e interesados para obtener una visión integral de las distintas posibilidades de desarrollo. Este mapa conceptual puede consultarse en la Figura 3 de este artículo.

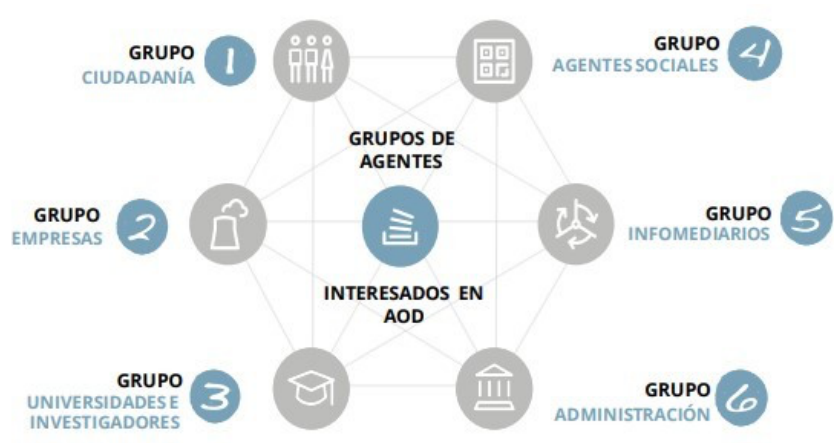

Figura 3. Mapa de agentes de Aragón Open Data

Con el objetivo de avanzar en su estrategia de datos abiertos, el Gobierno de Aragón ha considerado clave la participación para el buen funcionamiento de la iniciativa, y se han abierto canales donde los ciudadanos y empresas han podido trasladar sus necesidades en materia de datos. Con el fin de estimular las políticas de reutilización de la información del sector público, el Gobierno de Aragón trata de fomentar, mediante el impulso de iniciativas relevantes, el uso, reproducción, modificación o distribución de los conjuntos de datos.

Entre estas iniciativas destaca el proyecto Aragón Open Data Focus, lanzado en el año 2020, como una apuesta innovadora para ir un paso más allá en el desarrollo de los datos abiertos en el territorio. El objetivo principal del proyecto es construir un servicio que permita a cualquier persona crear narrativas digitales a partir de los datos abiertos disponibles en el catálogo de Aragón Open Data, poniendo a su disposición una herramienta que permita la reutilización de la informa- ción de forma efectiva y la generación colaborativa de conocimiento sobre la realidad económica, social, demográfica y cultural del territorio de una forma visual y atractiva.

De esta manera, Aragón Open Data Focus se presenta como una respuesta del Gobierno de Aragón al reto de acercar la utilidad de los datos abiertos a la ciudadanía, tejido empresarial y resto de agentes del territorio. Su implementación y desarrollo ha sido cofinanciada por el Fondo Europeo de Desarrollo Regional (FEDER) de la Unión Europea, cuyo lema es "Construyendo Europa desde Aragón".

\subsection{Contar historias con los datos como valor} diferencial de la apuesta de Aragón Open Data

Tal y como se ha adelantado, el proyecto Aragón Open Data Focus tiene su origen en la necesidad de mejorar el impacto del portal de datos abiertos Aragón Open Data. Para dar respuesta a este reto, se observaron distintas experiencias lanzadas en los últimos años en la esfera internacional en materia de reutilización de datos, identificándose como tendencia común el uso de visualizaciones para mejorar el entendimiento de la información que publican los poderes públicos. Entre estas experiencias analizadas destacan casos como Data USA (3), DataChile (4), Data Africa (5), el Departamento de Agricultura de EEUU (6) o el portal de datos abiertos de Singapur (7).

Los avances experimentados en los últimos años, tanto en accesibilidad y calidad de los datos como en el desarrollo de soluciones de tratamiento y visualización de estos, han provocado el surgimiento y auge del concepto data storytelling o "contar historias con los datos" como solución para la presentación, enriquecimiento y comprensión de la información en ámbitos tan diversos como la mejora de los procesos de toma de decisiones en el mundo empresarial (Suprata, 2019), la construcción de relatos publicitarios de marca (Caro-Castaño y Selva-Ruiz, 2019), la comunicación de análisis y resultados (Dykes, 2015) o el avance ético del periodismo (Lee et al., 2015).

La visualización de datos no es un elemento extraño a la estrategia de Aragón Open Data para avanzar en su labor sensibilización sobre el potencial de los datos abiertos: ya en 2018 desarrolló un servicio, Open Visual Data, que permitía a los usuarios del portal generar y utilizar sencillos gráficos con los datos disponibles en la web. Sin embargo, desde Gobierno de Aragón se ha llevado a cabo un esfuerzo adicional por configurar una propuesta de servicio un paso más allá, desarrollando para ello una herramienta de uso intuitivo que empodere a los usuarios menos 
avanzados del portal en el uso de los datos públicos para construir sus propias narrativas digitales, apostando por un uso innovador del open data más vinculado a las narrativas visuales (Segel y Heer, 2010) y el periodismo de datos (Flores y Salinas, 2013).

\section{Metodología del proyecto Aragón Open Data Focus}

El proyecto partió de una primera fase de análisis de los datos y servicios disponibles en el portal Aragón Open Data con el objetivo de conocer cuáles podrían ser los principales agentes interesados en cada uno de ellos, con el objetivo de mejorar la comunicación de su disponibilidad y facilitar su uso por parte de estos colectivos. Este diagnóstico de situación se estableció así como punto de partida que permitiera a futuro determinar la manera más efectiva y eficiente de aumentar el impacto del portal entre las distintas tipologías de agentes.

Así, en primer lugar, se definieron los distintos colectivos de potenciales usuarios del portal de Open Data de Aragón. Para ello, se examinaron distintas aproximaciones realizadas en otras iniciativas de datos abiertos en cuanto a la caracterización de los colectivos destinatarios de la información pública, identificándose como la práctica más interesante el trabajo desarrollado por la ciudad de Nueva York, Meet the Users of Open Data (NYC Open Data, 2017). Este trabajo define seis tipos de perfiles (Observador Ocupado, Explorador Equipado, Enlace Local, Intérprete Influyente, Mapeador Meticuloso y Campeón de la Comunidad) en función de su nivel de entendimiento de los datos abiertos, su actitud hacia ellos y su capacidad de influencia en la comunidad, y los caracteriza por sus habilidades en el uso de la tecnología, la recopilación y análisis de datos, la interpretación de información georreferenciada y el uso de los medios de comunicación. Los resultados de esta segmentación facilitan adaptar los servicios del portal, la oferta de servicios específica y la comunicación de todo ello a los distintos colectivos definidos.

A partir de este marco metodológico, y de forma alineada con la vocación establecida en la Estrategia Aragón Open Data, se definieron ocho tipos de colectivos de usuarios en el caso de Aragón, tal y como puede verse en la Figura 4.

Durante la fase inicial del proyecto, se trabajó en identificar el conocimiento y uso que los distintos colectivos identificados daban a las funcionalidades de los datos (informativo, consulta, explotación, descarga y visualización) del portal Aragón Open Data. De manera adicional, cada colectivo se caracterizó con base en una serie de atributos (entre ellos, las funcionalidades citadas para las cuales utilizaban la información y datos disponibles), caracterizando cada recurso de información disponible por estos atributos a su vez, dando lugar a un cruce de información que permitió evaluar la cantidad de información potencialmente reutilizable por cada uno de los colectivos, según los parámetros definidos. Los resultados de este trabajo se resumen en la Tabla II.

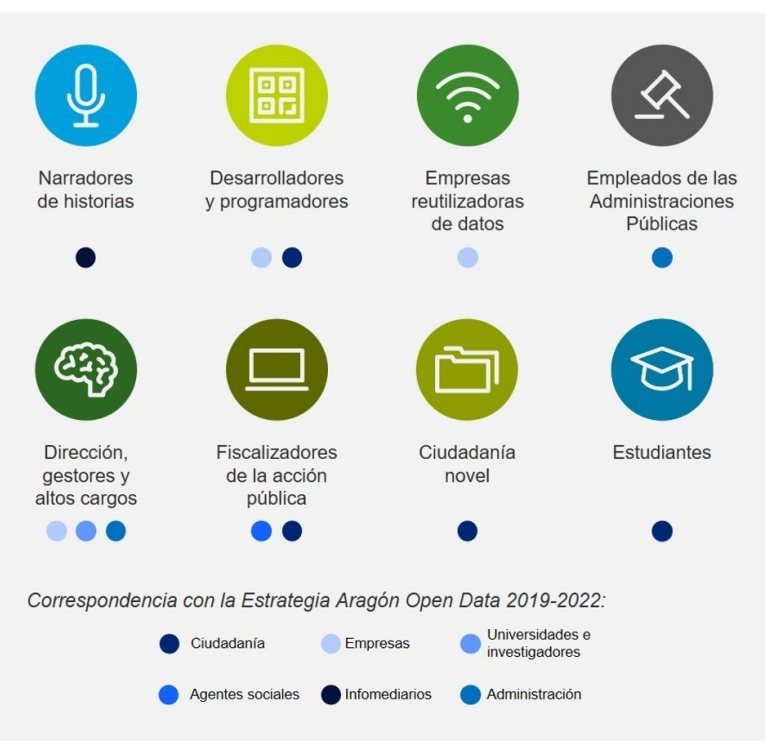

Figura 4. Colectivos de usuarios definidos en el marco del proyecto y correspondencia con los colectivos contemplados en la Estrategia Aragón Open Data 2019-2022

\begin{tabular}{lcc}
\hline $\begin{array}{l}\text { Colectivos de } \\
\text { usuarios }\end{array}$ & $\begin{array}{c}\text { Datasets con } \\
\text { potencial de uso }\end{array}$ & $\begin{array}{c}\text { \% de potencia- } \\
\text { lidad de uso }\end{array}$ \\
\hline $\begin{array}{l}\text { Narradores de } \\
\text { historias }\end{array}$ & 2.621 & $88 \%$ \\
\hline $\begin{array}{l}\text { Desarrolladores y } \\
\text { programadores }\end{array}$ & 1.955 & $66 \%$ \\
\hline $\begin{array}{l}\text { Empresas } \\
\text { reutilizadoras de } \\
\text { datos }\end{array}$ & 1.561 & $53 \%$ \\
\hline $\begin{array}{l}\text { Empleados de las } \\
\text { Administraciones } \\
\text { públicas }\end{array}$ & 2.735 & $92 \%$ \\
\hline $\begin{array}{l}\text { Dirección, } \\
\text { gestores y altos } \\
\text { cargos }\end{array}$ & & $21 \%$ \\
\hline $\begin{array}{l}\text { Fiscalizadores de } \\
\text { la acción pública }\end{array}$ & 647 & $22 \%$ \\
\hline \begin{tabular}{l} 
Ciudadania \\
\hline \begin{tabular}{l} 
Estudiantes \\
\hline
\end{tabular}
\end{tabular} & 647 & $14 \%$ \\
\hline
\end{tabular}

Tabla II. Colectivos de usuarios, conjuntos de datos disponibles por colectivo y potencialidad de uso 
Una vez realizado este diagnóstico, y con el fin de dar respuesta de la forma más adecuada a las necesidades de los usuarios, se inició un proceso de contraste con los distintos definidos. Para ello, se organizaron distintas mesas de trabajo temáticas en las que se establecieron debates en torno a los datos abiertos y se indagó en las expectativas y necesidades de estos distintos colectivos definidos.

Como consecuencia de este proceso de contraste se extrajeron una serie de conclusiones relativas a la accesibilidad de los datos, su reutilización y utilidad, para poder determinar si los mismos eran adecuados para dar respuesta a las necesidades reales de cada colectivo. En concreto, se observó la necesidad de fomentar la reutilización de datos por los distintos grupos de interés o colectivos de usuarios, formar a la comunidad de usuarios en materia de Open Data para que puedan extraer el mayor valor añadido posible de la plataforma, facilitar el uso y entendimiento del portal, e impulsar una comunidad de usuarios que interactúen y compartan sus conocimientos.

\section{Resultados}

\subsection{Arquitectura y funcionalidad} del nuevo servicio

A partir del trabajo realizado en la primera fase, comenzó a desarrollarse la herramienta que permitiría la creación de historias por parte de los usuarios. Dichas historias se basarán en datos disponibles en el portal de Open Data del Gobierno de Aragón, de manera que los usuarios puedan construir un relato atractivo y diferencial en torno a un conjunto de datos disponible, otorgándoles un contexto y una interpretación que pueda ser de interés para el resto.

En este sentido, la plataforma, permitirá a los usuarios observar y leer las historias generadas por otros y realizar aportaciones o contribuciones a las mismas, de manera que se fomente un espacio de co-creación y colaboración entre distintos usuarios. El objetivo, será el de crear un portal que albergue de manera progresiva las historias generadas por los distintos grupos y colectivos de usuarios, contadas a través de datos abiertos.

La implementación del nuevo servicio se ha llevado a cabo en la arquitectura actual de entornos de la plataforma Aragón Open Data, si bien se han producido modificaciones en algunos de los componentes para la configuración de Aragón Open Data Focus:
1. Open Data Focus: La tecnología utilizada para el frontend de Open Data Focus es Angular. Reutilizando el proyecto base de Open Visual Data, se ha creado un servicio como un portal nuevo, dejando el anterior servicio como mera herramienta.

2. Open Visual Data: El proyecto de Open Visual Data, con ese mismo nombre, quedará descontinuado en su repositorio de código. Mientras tanto, la nueva herramienta de creación de gráficos de Aragón Open Data vivirá dentro del nuevo proyecto Open Data Focus, utilizando la misma tecnología, Angular.

3. Focus API: El proyecto Open Data Focus necesita de una potente API que interactúe con la base de datos, para ello se reutiliza la API de Open Visual Data, desarrollada en Spring Boot y se nutre de nuevas funcionalidades, se mejora lo que ya había y se condiciona para cumplir los requisitos del proyecto.

4. Visual Data API: El proyecto Open Visual Data, contenía una API que quedará descontinuada en su repositorio de código.

5. Update Charts: Es un proceso NodeJS, que se ejecuta diariamente y actualiza todas las gráficas disponibles en la plataforma.

6. Base de datos: Utilizando la tecnología MongoDB, se mantiene la misma base de datos que se utilizaba para el proyecto de Open Visual Data. Tan solo sufre algunos cambios de estructura debido a las nuevas funcionalidades y al proceso interno de actualización diario de las gráficas.

Con el objetivo de mantener la homogeneidad en la infraestructura del proyecto de Aragón Open Data, las tecnologías utilizadas para este desarrollo han sido NodeJS para el backend y servicios web, Angular para la parte frontal y visual del portal, y PostgreSQL como soporte de almacenamiento de datos.

A nivel de funcionalidades, el usuario será capaz de interactuar con la parte visual de Aragón Open Data Focus (Angular), gracias a un navegador web. Todas las peticiones que realice el usuario serán recogidas a través de la lógica de Angular, que llamará a su vez a la API localizada en el backend (NodeJS) en continuo estado de escucha. La API (NodeJS) se encargará de transformar estas peticiones del usuario en consultas a la base de datos (PostgreSQL), que devolverá los resultados pedidos con el objetivo de que el frontal (Angular) sea capaz de mostrarlos en forma de historia.

De cara a la utilización del servicio, se ha llevado a cabo un esfuerzo específico para garantizar un 
uso lo más sencillo e intuitivo posible, con una interfaz única independientemente del tipo de usuario que haga uso del servicio, al que no se le solicita ningún dato personal para poder utilizar el servicio, en línea con los principios rectores de los datos abiertos que propugnan una accesibilidad total de los mismos. Al acceder a Aragón Open Data Focus, el usuario se encuentra ante una pantalla dividida en cuatro secciones: título de la historia, descripción (con un máximo de 420 caracteres), categorización (que permite añadir una o varias categorías a la historia para facilitar la búsqueda de textos relacionados en función de las temáticas definidas) y secciones. Puede verse una de las funcionalidades descritas en la Figura 5.

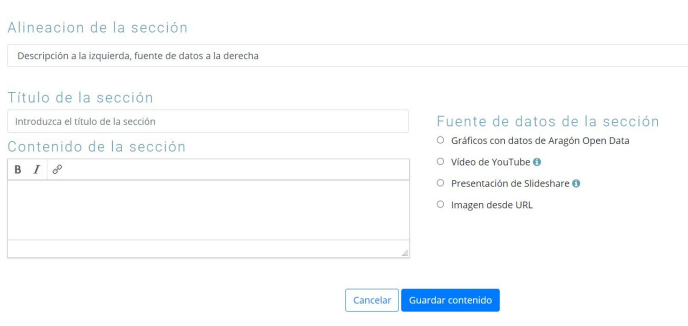

Figura 5. Funcionalidad "Añadir sección"

En cada una de las secciones, el usuario de Aragón Open Data puede incluir distintos recursos que le permitan reforzar los mensajes que compongan su texto: desde vídeos de la plataforma YouTube hasta presentaciones de SlideShare o imágenes publicadas en Internet. En esta funcionalidad es donde destaca la posibilidad de crear gráficos a partir de los recursos del banco de datos de Aragón Open Data, ya sean visualizaciones preexistentes $u$ otras nuevas a partir de los datos abiertos del portal.

Así, el proceso de co-creación queda configurado de la siguiente manera: el Gobierno de Aragón publica y pone a disposición de cualquier usuario los datos a través del portal Aragón Open Data, y provee este servicio que permite el tratamiento de los datos de forma sencilla a través de la propia web, siendo los usuarios los que deciden cómo explotan estos datos para respaldar sus propias historias. Adicionalmente, y como ya se ha indicado, si bien se ha trabajado en que el servicio web sea lo más intuitivo posible, también se ha creado un vídeo tutorial (8), accesible a través de la web y alojado en YouTube, para facilitar la comprensión y uso del servicio, si bien también pueden realizarse preguntas más concretas a través del correo electrónico opendata@aragon.es. Una vez finalizada la historia, es moderada por el propio equipo responsable del portal, si bien esta mo- deración se limita a la corrección gramatical y ortográfica de los textos presentados y a que el contenido no sea inadecuado (por ejemplo, referido a actividades ilícitas, como el racismo, la xenofobia o la apología del terrorismo, o nocivos, peligrosos o poco saludables) y no infrinja las leyes de protección de datos o propiedad intelectual.

Adicionalmente, y con el objetivo de capacitar a los usuarios y dar a conocer la plataforma, se ha creado una formación ad hoc sobre Aragón Open Data Focus y cómo contar historias con datos que capacitará a los empleados de la Administración pública en esta primera fase del despliegue del nuevo servicio.

\subsection{Aragón Open Data Focus: las historias al servicio de los datos}

Como consecuencia de los trabajos realizados con los distintos colectivos de usuarios consultados, se identificaron mejoras diversas a realizar en el portal de Aragón Open Data. Adicionalmente, en relación con el servicio Aragón Open Data Focus (9), se perfilaron diversas temáticas acerca de las cuales sería de interés para los usuarios que se escribiera una historia, como, por ejemplo, los alojamientos turísticos rurales disponibles en Aragón, un análisis del gasto que se destina a la educación en el territorio o el avance de Aragón en su transformación hacia una sociedad de la información. Así, con el servicio recién estrenado, ya están disponibles algunas historias acerca de las temáticas consideradas de interés. A modo de ejemplo se presentan las siguientes:

- Destaca en primer lugar, por su sentido de actualidad, la historia "El coronavirus en Aragón" que relata la incidencia de la COVID-19 en el territorio y su evolución. La historia cuenta, basándose en los datos disponibles, cómo Zaragoza ha sido la más damnificada de las provincias de la comunidad autónoma, concentrando 6 de cada 10 casos confirmados en la región.

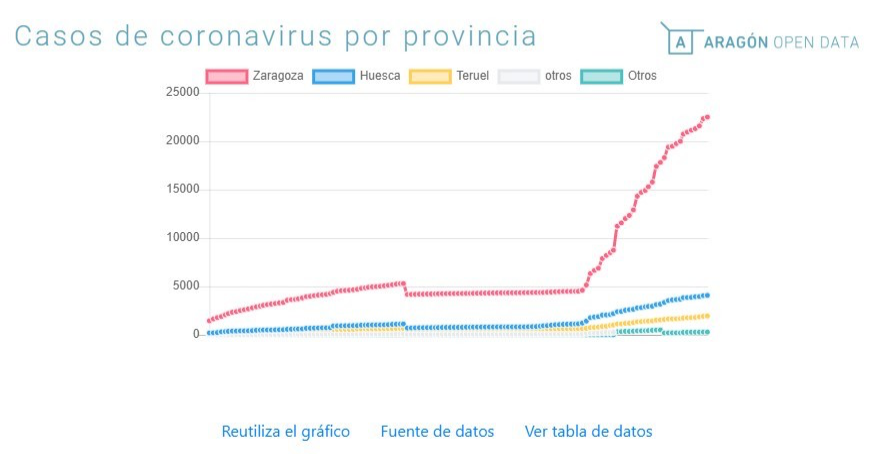

Figura 6. Historia "El coronavirus en Aragón" 
Asimismo, la historia ofrece la visualización gráfica de la curva de la pandemia en el territorio y las distintas medidas que van a ser adoptadas para paliar las consecuencias, así como los fondos económicos destinados a la reconstrucción de la región o el rol del sistema aragonés de salud en la lucha contra la pandemia. Un ejemplo visual de los gráficos creados para esta historia puede consultarse en la Figura 6.

- Asimismo, destaca la historia que analiza el impacto de la COVID-19 en el turismo aragonés, "Este verano salimos juntos en Aragón", que trata de poner en valor el atractivo paisajístico, cultural y gastronómico que ofrece el territorio aragonés basándose en datos e información pública, tratando de incentivar el turismo interior en una época en que se recomienda evitar los desplazamientos en la medida de lo posible. La historia analiza los datos relativos a la oferta de plazas hoteleras y cuenta los atractivos culturales existentes en el entorno de Zaragoza y las oportunidades de turismo activo o rural y gastronómico que ofrece el entorno de Huesca, con el objetivo de atraer turistas locales y de otras comunidades autónomas.

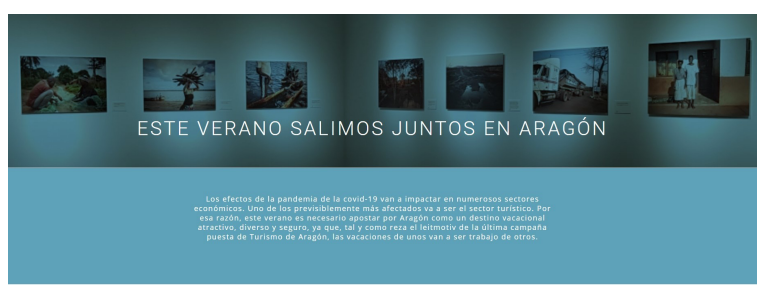

Figura 7. Historia "Este verano salimos juntos en Aragón"

- Otra de las historias destaca el impacto de la COVID-19 en el colectivo universitario del teritorio, y, específicamente, cómo la pandemia ha afectado a la movilidad de los estudiantes universitarios. Basándose en los datos históricos relativos a los acuerdos Erasmus y SICUE de los estudiantes de la Universidad de Zaragoza, se analizan los destinos favoritos de los estudiantes, resultando como destinos más atractivos Granada, a nivel nacional, e Italia, a nivel internacional, pudiendo verse afectada la tendencia de cursar estudios fuera en el presente curso. Asimismo, la historia trata de relatar cómo ha evolucionado la solicitud para cursar determinados estudios por parte del alumnado, habiendo ganado relevancia todo lo relativo a los grados y titulaciones de la rama biosanitaria y los grados de carácter más técnico, como matemáticas o física. Por último, la historia pone en contexto el dato relativo a las ayudas o becas a estudiantes, reforzando la idea de que la COVID19 no puede ser un impedimento para el desarrollo formativo adecuado de la juventud aragonesa.

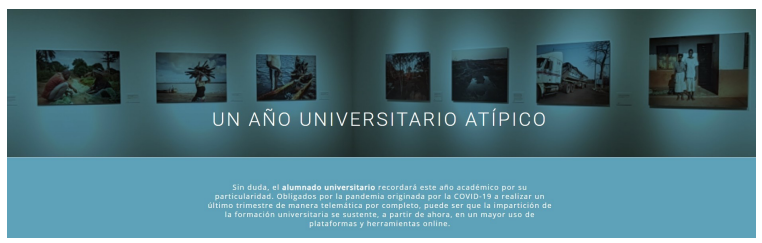

Figura 8. Historia "Un año universitario atípico"

- Además de las historias inspiradas por la pandemia, se han dibujado otras, como la historia "El avance de Aragón en su transformación hacia una sociedad de la información", que trata de relatar los avances que se han dado en los últimos años en materia TIC y cómo estos han contribuido a la transformación de la sociedad aragonesa. El artículo pone de manifiesto el contexto caracterizado por la progresiva importancia de las TICs en las distintas vertientes de la vida, ocupando un rol fundamental en la mejora de la competitividad del territorio. En este contexto, se habla del esfuerzo conjunto realizado por parte del Gobierno, empresas y sociedad de Aragón para convertirse en una de las denominadas "regiones inteligentes", utilizando para ello los datos relativos a la incorporación de profesionales expertos en las TIC en las empresas del territorio o la disponibilidad de ordenadores en determinados sectores de actividad.

\section{Conclusiones}

El nuevo servicio del proyecto de Aragón Open Data, Aragón Open Data Focus, se constituye como una experiencia innovadora y pionera a nivel estatal en materia de reutilización de datos del sector público. Este servicio permite a los usuarios del portal utilizar los datos abiertos del catálogo de Aragón Open Data para elaborar sus propias historias basadas en ellos y construir visualizaciones que faciliten o enriquezcan la comprensión de los lectores de la información del sector público.

Si bien aun es pronto para poder medir el éxito del proyecto, que vendrá determinado por el aumento del uso y reutilización de los datos y la mejora de la capacitación de los distintos colectivos de usuarios para reutilizar la información pública, pueden ponerse en valor algunos indicios alenta- 
dores del impacto buscado por el equipo de la Dirección General de Administración Electrónica y Sociedad de la Información de Gobierno de Aragón: por un lado, destaca la adhesión e interés que ha suscitado este proyecto durante su desarrollo, con casi 300 participantes asistiendo a los distintos eventos online celebrados en los meses de mayo a septiembre de 2020; por otro, el uso que se está haciendo del servicio, con 14 historias publicadas a mayo de 2021. Estas primeras historias se espera que se conviertan en la primera piedra del proyecto de construcción de una comunidad empoderada de usuarios, activa y comprometida, que alimente el portal web con nuevas narrativas basadas en los datos abiertos del territorio, que sea capaz de generar un diálogo en torno a estos datos y que permita avanzar, a la postre, hacia un modelo de reutilización más intensiva de los datos abiertos de Aragón.

Así, a futuro será muy relevante que los datos disponibles sigan creciendo y mejorando su calidad para que los usuarios puedan disponer de información relevante, veraz y actualizada, y que las historias que se cuelguen en la plataforma puedan cambiar y evolucionar a la vez que los datos. La actualización de los datos contribuirá a nuevas interpretaciones y relatos vivos sobre distintos ámbitos y cuestiones que afectan al territorio, dando un valor diferencial a la información, ya que los datos en sí mismos, sin contexto e interpretación, carecen de valor.

\section{Notas}

(1) https://opendata.aragon.es/

(2) https://opendata.aragon.es/datos/catalogo

(3) https://datausa.io/

(4) https://es.datachile.io/

(5) https://dataafrica.io/

(6) https://www.ers.usda.gov/data-products/data-visualizations/

(7) https://data.gov.sg/

(8) https://www.youtube.com/watch?v=AnNnt6weWx4\&ab channel=ARAGONOPENDATA

(9) https://opendata.aragon.es/servicios/focus/

\section{Referencias}

Abella, Alberto. (2019). La reutilización de datos abiertos en España II. desideDatum. https://www.desidedatum.com/ wp-content/uploads/2019/12/La_reutilizacio\%CC\%81n_ datos_abiertos_en_espan\%CC\%83a_2019.pdf

Aleixandre-Benavent, Rafael; Ferrer Sapena, Antonia; Peset, Fernanda. (2019). Compartir los recursos útiles para la investigación: datos abiertos (open data). // Educación Médica, ISSN: 1575-1813. Elsevier. 22:2. DOI: 10.1016/j.edumed.2019.07.004
Álvarez García, Sergio; Gértrudix Barrio, Manuel; Rajas Fernández, Mario. (2014). La construcción colaborativa de bancos de datos abiertos como instrumento de empoderamiento ciudadano. // Revista Latina de Comunicación Social. 69:6, 661-683. Universidad de La Laguna. http://www.revistalatinacs.org/069/paper/1029_UR/RL CS_paper1029.pdf

Attard, Judie; Orlandi, Fabrizio; Scerri, Simon; Auer, Sören (2015). A systematic review of open government data initiatives. // Government Information Quarterly. 32:4, 399418. https://www.sciencedirect.com/science/article/abs/ pii/S0740624X1500091X?via\%3Dihub

Bichard, Jo-Anne; Knight, Gail (2012) Improving public services through open data: public toilets. // Municipal Engineer. 165:ME3, 157-165. https://researchonline.rca.ac. uk/1119/1/Bichard\%20\&\%20Knight.pdf

Blanes Climent, Miguel Ángel (2013). La transparencia informativa de las administraciones públicas: el derecho de las personas a saber y la obligación de difundir información pública de forma activa. Universidad de Alicante. http://rua.ua.es/dspace/bitstream/10045/35719/3/tesis_ miguelangel_blanescliment.pdf

Cabezali, Pablo. (2020) Diagnóstico de los datos abiertos en España, evaluación de los portales autonómicos de datos abiertos y desarrollo de cuadros de mando para la exposición de resultados. Universidad de Deusto. https://dkh.deusto.es/comunidad/explorer/recurso/diagnostico-de-los-datos-abiertos-en-espaa/54b38648-d6054974-a441-7d869e020391

Caro-Castaño, Lucía; Selva-Ruiz, David (2020). Data storytelling: el empleo de datos abiertos en la construcción de relatos publicitarios de marca. // Comunicación y hombre: revista interdisciplinar de ciencias de la comunicación y humanidades. 16, 35-57. Universidad Francisco de Vitoria. https://portalderevistas.ufv.es/index.php/comunicacio nyhombre/article/view/572/585

Cerrillo i Martínez, Agustí (2011). Transparencia administrativa y lucha contra la corrupción en la Administración local. // Anuario del Gobierno Local. 1, 277-313. http://repositorio.gobiernolocal.es/xmlui/bitstream/handle/10873/ 1255/16_Cerrillo_Transparencia.pdf?sequence=1\&isAllowed $=\mathrm{y}$

Clabo, Néstor; Ramos-Vielba, Irene (2015). Reutilización de datos abiertos en la administración pública en España y uso de licencias-tipo. // Revista Española de Documentación Científica. 38:3, e097. http://redc.revistas.csic.es/index.php/redc/article/view/900/1269

Conejero Paz, Enrique (2013). Gobierno abierto y democracia participativa. // 3c Empresa: investigación y pensamiento crítico. 2:4, 2013. https://dialnet.unirioja.es/descarga/articulo/4817923.pdf

Cordero Sanz, Carlos (2007). Derecho de acceso a la información y transparencia: herramientas necesarias para la democracia en una sociedad abierta. // El derecho de acceso a la información pública, 55-67. https://eprints.ucm.es/id/eprint/8946/1/MANUEL_SANCHEZ_DER_ ACCESO_V14_2.pdf

Cotino Hueso, Lorenzo (2015). La nueva Ley de transparencia y acceso a la información. // Anuario de la Facultad de Derecho. 241-256. https://ebuah.uah.es/dspace/bits tream/handle/10017/22075/nueva_cotino_AFDUA 2014. pdf?sequence $=1$ \&isAllowed $=y$

Dykes, Brent (2015). Data storytelling: What it is and how it can be used to effectively communicate analysis results. // Applied Marketing Analytics. 1, Issue 4. HSTalks. https://hstalks.com/article/619/data-storytelling-what-it-isand-how-it-can-be-use/ 
European Data Portal (2020). The Economic Impact of Open Data: Opportunities for value creation in Europe. ISBN: 978-92-78-42108-3 European Commission. https://www.europeandataportal.eu/es/highlights/theeconomic-impact-of-open-data

European Data Portal (2020). Analytical Report 12: Businessto-Government Data Sharing. ISBN: 978-92-78-41997-4. European Commission. https://data.europa.eu/sites/default/files/analytical_report_12_business_government_data_sharing.pdf

Flores Vivar, Jesús; Salinas, Aguilar, Cecilia. (2013). El periodismo de datos como especialización de las organizaciones de noticias en Internet. // Correspondencias y Análisis. 3, 15-34. https://dialnet.unirioja.es/descarga/articulo/ 4739290.pdf

Gobierno de Aragón (2019). Estrategia Aragón Open Data [2019-2022]. https://opendata.aragon.es/static/public/documentos/20190405_estrategia_opendata.pdf

Greed, Clara (2003). Inclusive Urban Design: Public Toilets. Routledge.

Kucera, Jan; Chlapek, Dusan (2014). Benefits and Risks of Open Government Data. // Journal of Systems Integration. ISSN: 1804-2724. 5:1. http://si-journal.org/index. php/JSI/article/viewFile/185/254

Lee, Bongshing; Riche, Nathalie Henry; Isenberg, Petra; Carpendale, Sheelagh (2015). More than telling a story: transforming data into visually shared stories. // IEEE Computer Graphics and Applications. 35:5. https://www.microsoft.com/en-us/research/uploads/prod/ 2016/12/StorytellingProcess-CGA2015.pdf

Montero Caro, María Dolores (2020). El encaje constitucional del modelo de Open Government. Universidad de Córdoba: UCOPRess. https://helvia.uco.es/xmlui/handle/ 10396/19485

NYC Open Data (2017). Meet the Users of Open Data. https://thereboot.github.io/moti-personas/
Obama, Barack (2009). Transparency and Open Government Memorandum. https://obamawhitehouse.archives.gov/ the-press-office/transparency-and-open-government

Open Knowledge Foundation (2012). Open Data Handbook. https://opendatahandbook.org/guide/es/what-is-opendatal

Palomares Herrera, Manuel (2017). Estudio comparado sobre transparencia y derecho de acceso en el ámbito internacional y su influencia en España. // lus Humani: Revista de Derecho. 6, 123-153. https://dialnet.unirioja.es/descarga/articulo/5877166.pdf

Ramos Simón, L. Fernando; Arquero Avilés, Rosario; Botezan, Iuliana; Cobo Serrano, Silvia; Sala Jiménez, Andrea; Sánchez Jiménez, Rodrigo; Del Valle Gastamiza; Félix (2012). De la reutilización de información del sector público a los portales de datos abiertos en Europa. // BiD: Textos universitaris de biblioteconomia y documentació. 29. http://bid.ub.edu/29/ramos2.htm

Segel, Edward; Heer, Jeffrey (2010). Narrative Visualization: Telling Stories with Data. // IEEE Transactions on Visualization and Computer Graphics. 16:6. https://ieeexplore.ieee.org/document/5613452

Sève, Bruno; Redondo, Ernesto (2019). El pabellón de deseos. Co-creación y co-instalación artística para la mejora del espacio público. ACE: Architecture, City and Environment. 14:42, 8200. https://upcommons.upc.edu/bit stream/handle/2117/178902/8200-8741-1-PB.pdf?sequence $=1$

Suprata, Ferdian (2019). Data Storytelling with Dashboard: Accelerating Understanding through Data Visualization in Financial Technology Company Case Study. // Journal Metris. 20:1. http://ojs.atmajaya.ac.id/index.php/metris/ article/view/1441/1047.

Enviado: 2021-05-11. Segunda versión: 2021-05-31. Aceptado: 2021-06-16. 
